\title{
The uneasy coexistence of predators and pathogens ${ }^{\star}$
}

\author{
Andreas Eilersen $^{\mathrm{a}}$ and Kim Sneppen ${ }^{\mathrm{b}}$ \\ Niels Bohr Institute, University of Copenhagen, Blegdamsvej 17, 2100 København Ø, Denmark
}

Received 28 February 2020 and Received in final form 19 May 2020

Published online: 2 July 2020

(C) The Author(s) 2020. This article is published with open access at Springerlink.com

\begin{abstract}
Disease and predation are both highly important in ecology, and pathogens with multiple host species have turned out to be common. Nonetheless, the interplay between multi-host epidemics and predation has received relatively little attention. Here, we analyse a model of a predator-prey system with disease in both prey and predator populations and determine reasonable parameter values using allometric mass scaling relations. Our analysis focuses on the possibility of extinction events rather than the linear stability of the model equations, and we derive approximate relations for the parameter values at which we expect these events to occur. We find that if the predator is a specialist, epidemics frequently drive the predator species to extinction. If the predator has an additional, immune prey species, predators will usually survive. Coexistence of predator and disease is impossible in the single-prey model. We conclude that for the prey species, carrying a pathogen can be an effective weapon against predators, and that being a generalist is a major advantage for a predator in the event of an epidemic affecting the prey or both species.
\end{abstract}

\section{Introduction}

Predation is one of the fundamental modes of interaction among living organisms. Mechanisms similar to predation are found in anything from mammals to bacteria. Another equally important factor is epidemic disease, which is also found on all scales in the ecosphere. In recent years it has become clear that many epidemic pathogens are shared between several species [1], of which some presumably prey on each other. If the predator runs a risk of becoming infected when eating infected prey, it is possible that the prey species will be able to use the pathogen as a weapon against the predator. This could even be a very effective evolutionary strategy, given that prey species are often much more numerous than their predators, leading to a high infection pressure against the predator species [2]. On this basis, we propose the hypothesis that a disease shared between a prey species and its predator will turn out to be a major problem for the predator, and thus perhaps a long-term advantage for the prey. However, if the predator has several prey options, epidemics should pose much less of a threat to it, as it can just feed on an immune prey species in the event of an epidemic.

\footnotetext{
* Supplementary material in the form of a .pdf file available from the Journal web page at

https://doi.org/10.1140/epje/i2020-11966-7

a e-mail: andreaseilersen@nbi.ku.dk

b e-mail: sneppen@nbi.ku.dk
}

The dynamics of predator-prey-pathogen interactions in general have received some attention in recent decades. Most attention has been given to the interaction between predators and single-host epidemics or parasitism [3-7]. Lafferty et al. [8] have attempted to coordinate the variations on Lotka-Volterra and SIR models that have been proposed to deal with predation, disease, and parasitism. In their work, they propose a general framework underlying all consumer-resource models. However, the interplay between predation and multi-host infectious disease specifically has not been as thoroughly studied. Though this is partly justified by the similarity between parasitism and multi-host epidemic disease, the pathogen that we here want to study lacks the life-cycle dynamics associated with trophically transmitted parasites [8].

A few models similar to the one we will put forward in this paper do exist. Hsieh and Hsiao [9] have constructed one such model, and Han et al. [10] briefly cover another. These examples focus their analyses on the linear stability of the fixed points of their system, whereas we will focus on extinction events. We choose this focus, since an epidemic outbreak at least initially is a perturbation away from equilibrium which may temporarily drive populations to such low densities that it would lead to extinction in the real world. We will attempt to derive analytical relations for the boundaries between predator-, disease-, and prey-dominated regions of parameter space.

When analysing epidemiological models, it is difficult but crucial to determine what parameter ranges are 
reasonable. A discussion of this problem is often missing from more theoretical treatments $[9,10]$. Therefore, we will here attempt to use the allometric mass scaling laws for many demographic and epidemiological quantities to estimate the range of parameters.

It has long been known that quantities such as reproduction rate and metabolic effect scale with animal mass to some quarter power [11]. Attempts have been made in ecology to use this to predict the behaviour of predatorprey systems [12-14]. More recently, it has been shown that disease recovery and death rates also scale with animal mass [15], which is useful in epidemiological modelling [16]. The parameterisation that we will use here will be based in part on our previous work on parameterising the Lotka-Volterra predator-prey equations [14]. The mass scaling relations are for the most part fairly general across different classes of animals. We will here be using the mass scaling relations valid for mammals. One could construct similar models for predation among other animals by mainly changing the constants of proportionality [11], and we would therefore expect our model to be relevant even for non-mammals. Only when looking at entirely different organisms such as bacteria do we need to be more careful, as the mechanisms that might be responsible for the scaling are different [17]. Nonetheless, a similar scaling law for metabolic effect exists even for bacteria [18].

In summary, the questions that we will try to answer here will be whether an epidemic affecting a prey species can drive a predator species to extinction, and if so, for what parameter values this will be most likely. We also want to examine the effect of a predator being a generalist, i.e. having an alternative prey option that is not affected by the epidemic.

\section{The model}

To study this system, we use the Rosenzweig-MacArthur equations for predator-prey interactions [19], but with a modified predator death rate:

$$
\begin{aligned}
& \frac{\mathrm{d} x}{\mathrm{~d} t}=\alpha x(1-x / K)-\phi x \frac{y}{x+\epsilon}, \\
& \frac{\mathrm{d} y}{\mathrm{~d} t}=\nu y \frac{x}{x+\epsilon}-\delta y \frac{\epsilon}{x+\epsilon},
\end{aligned}
$$

where $x$ is prey, $y$ is predator, $\alpha$ is the per capita prey reproduction rate and $\delta$ is the predator starvation rate in the absence of prey [20]. $K$ is the prey carrying capacity and $\epsilon$ is the half-saturation constant for predators. $\phi$ and $\nu$ are coupling constants of the functional and numerical responses, respectively [21]. We choose to modify the predator death rate, since we do not expect this rate to be constant with respect to prey population. Instead, it should approach $\delta$ when there is no prey and the predators starve at a constant rate, and zero if there is a lot of prey. The last part holds if starvation is assumed to be the primary cause of death for predators, as in the LotkaVolterra model [20].
We will combine these equations with the SIR model, which gives the following equations for the changes in population during an epidemic:

$$
\begin{aligned}
\frac{\mathrm{d} S}{\mathrm{~d} t} & =-\beta S I, \\
\frac{\mathrm{d} I}{\mathrm{~d} t} & =\beta S I-\gamma I, \\
\frac{\mathrm{d} R}{\mathrm{~d} t} & =\gamma I .
\end{aligned}
$$

Here, $S$ denotes susceptible individuals, $I$ infected, and $R$ recovered or dead individuals. $\beta$ gives the rate at which each infected individual infects susceptible individuals, and $\gamma$ gives the death or recovery rate of the infected [22].

When constructing our model, we shall make the assumption that the disease is always deadly, as the possibility of recovery with immunity will vastly complicate the analysis in a predator-prey system. Furthermore, we assume that infection from predator to prey is impossible, as any close encounters between the two species are likely to cause the immediate death of the prey. When modelling the system below, we find that varying the predatorpredator infection rate makes relatively little difference. Figures illustrating this can be found in the supplement. For the sake of simplicity, in the following we will therefore only treat the case where the majority of predator infections stem from prey, and predator-predator infections can be neglected. We also let only healthy animals reproduce, although both healthy and infected predators eat prey. Combining the SIR and Rosenzweig-MacArthur models, we end up with the following equations for the single-prey system:

$$
\begin{aligned}
\frac{\mathrm{d} S_{x}}{\mathrm{~d} t}= & \alpha S_{x}\left(1-\left(S_{x}+I_{x}\right) / K\right) \\
& -\beta_{x x} S_{x} I_{x}-\phi \frac{S_{y}+I_{y}}{S_{x}+I_{x}+\epsilon} S_{x} \\
\frac{\mathrm{d} I_{x}}{\mathrm{~d} t}= & \beta_{x x} S_{x} I_{x}-\phi \frac{S_{y}+I_{y}}{S_{x}+I_{x}+\epsilon} I_{x}-\gamma_{x} I_{x} \\
\frac{\mathrm{d} S_{y}}{\mathrm{~d} t}= & \nu \frac{S_{x}+I_{x}}{S_{x}+I_{x}+\epsilon} S_{y}-\beta_{y x} S_{y} I_{x}-\delta \frac{\epsilon}{S_{x}+I_{x}+\epsilon} S_{y} \\
\frac{\mathrm{d} I_{y}}{\mathrm{~d} t}= & \beta_{y x} S_{y} I_{x}-\gamma_{y} I_{y}-\delta \frac{\epsilon}{S_{x}+I_{x}+\epsilon} I_{y} .
\end{aligned}
$$

The equations for the number of dead individuals have been dropped, as they add no information when the disease is universally fatal. Subscripts here denote the species, with $\beta_{i j}$ being the coefficient for infection from species $j$ to species $i$. If we set the probability of infection when eating an infected prey equal to 1 , the infection coefficient $\beta_{y x}$ becomes equal to $\frac{\phi}{S_{x}+I_{x}+\epsilon}$, as the number of infected prey eaten equals the number of predators infected.

It should be noted that the exact functional form of these equations is not very important for the conclusions of this study. In the Electronic Supplementary Material, we show a parameter sweep analogous to the one found in fig. 1, but using the classical Lotka-Volterra equations 
with linear functional and numerical responses. This turns out to not significantly affect the conclusions of this study.

From $[11,14,23]$ we can find relations between predator and prey mass $\left(m_{x}\right.$ and $\left.m_{y}\right)$ and the parameters $\alpha, \delta, \phi$, and $\nu$. We want $\alpha$ and $\nu$ to represent theoretical maximal reproduction rates for prey and predators respectively. Instead of using the data from growing populations in the wild, where starvation, disease and other complications practically always play a role, we believe that the theoretical cap on reproduction should be set by the gestation period. $\alpha$ and $\nu$ should thus be the inverse gestation pe$\operatorname{riod}[11]$ :

$$
\alpha \approx 1 / t_{g} \approx \frac{1}{50} m_{x}^{-1 / 4}, \quad \nu \approx \frac{1}{50} m_{y}^{-1 / 4} \quad[1 / \text { days }],
$$

with mass in kilograms. A similar mass scaling law can be found for the incubation period of species that lay eggs [24]. We assume that when the predator is satisfied $\left(S_{y} \approx \epsilon\right)$, the predator population is constant, giving us $\delta \approx \frac{1}{50} m_{y}^{-1 / 4}$ as well. In order to calculate how many prey the predators need to eat to reproduce this much, we must know the ecological efficiency $\eta$. The ecological efficiency, defined as the fraction of consumed prey biomass converted into predator biomass, we estimate to be $10 \%$ although the quantity varies significantly with trophic level and the specifics of the species $[25,26]$. Knowing the efficiency, we can calculate the number of prey eaten as $\phi \frac{S_{x}}{S_{x}+\epsilon} S_{y}=\frac{m_{y}}{\eta m_{x}} \nu \frac{S_{y}}{S_{x}+\epsilon} S_{x}$, which implies $\phi=\frac{10 m_{y}}{m_{x}} \nu=\frac{m_{y}^{3 / 4}}{5 m_{x}}$ Finally, also from Peters [11], we have the following approximate relation for the carrying capacity:

$$
K \approx 200 m_{x}^{-3 / 4} \quad\left[\text { prey } / \mathrm{km}^{2}\right] .
$$

This relation is valid if we assume that the prey is a mammal and accept that the metabolic scaling exponent $3 / 4$ is the "true" theoretical value of the empirically estimated scaling exponent $(\sim 0.61)$ of the carrying capacity. By using this carrying capacity relation, we decide that the units of the population densities are $\left[\mathrm{km}^{-2}\right] . \epsilon$ is difficult to determine, and we therefore choose to set $\epsilon=K / 2$. We believe this to be reasonable, as it allows the predator population growth to saturate before the prey population reaches its carrying capacity. However, as can be seen in the supplement, we can set $\epsilon$ to practically any value between $0.3 K$ and $K$ and still get similar results.

To extend the predator-prey model to the predatorprey-disease case, we also need to know the scaling relations for disease duration. According to Cable et al. [15], both the time until first symptoms and the time until recovery or death scale as $t=\mathrm{cm}^{1 / 4}$, where $c$ is an experimental constant. Here, we shall use the constants appropriate for rabies. We choose to use these constants since we need an estimate of the order of magnitude of the scaling coefficient. It should be stressed, however, that the disease modelled here does not correspond to any single real-world disease, since we also wish to study the effects of varying its infectivity.

According to Cable et al. the duration of the period during which the infected individual is symptomatic can be written $t_{I} \approx t_{D}-t_{S}=\left(c_{2}-c_{1}\right) m^{1 / 4}$, where $c_{1}$ and $c_{2}$ are the scaling coefficients appropriate for the time until first symptoms and death, respectively. We assume that this period is of the same order as the infective period of the disease. The constants have been determined using statistical analysis, and their values are $c_{1}=9(4,19)$ and $c_{2}=16(7,32)$, where the numbers in parentheses are the boundaries of the confidence interval from $p=2.5 \%$ to $p=97.5 \%[15,27] . \gamma_{i}$ can now be found as $1 / t_{I, i}$.

Finally, to make the parameterisation more intuitive, we choose to express infectivity in terms of a quantity $R_{x x}$ related to the basic reproduction number $\left(R_{0}\right)$ of the disease. The basic reproduction number represents the number of secondary infections that occur when exposing an infected individual to a completely susceptible population. The reproduction number is related to the infection coefficient as $R_{i j}=\frac{\beta_{i j} S_{i, 0}}{\gamma_{j}}$ in the SIR model [2], where $S_{i, 0}$ is the initial density of susceptible individuals of species $i$ at the onset of the epidemic. $R_{0}$ has the important property that if it is less than 1 , the disease-free equilibrium is stable in the SIR model. We find that for the prey-prey reproduction number $R_{x x}$ to have this property in the case with immune predators, we cannot simply use the formula given above. Instead, we would have to add a correction, so that $R_{x x} \rightarrow R_{x x}-\frac{\alpha}{\gamma_{x}}\left(1-\frac{\delta \epsilon}{\nu K}\right)$. Nonetheless, for simplicity we will here use the formula $R_{x x}=\frac{\beta_{x x} S_{x, 0}}{\gamma_{x}}$, since the correction is quite small $(\sim 0.1)$ for most parameter values in our parameterisation. If we choose the starting population $S_{x, 0}$ to be the predator-prey equilibrium in the absence of disease, we have $R_{x x}=\frac{\beta_{x x} \delta \epsilon}{\gamma_{x} \nu}$.

$R_{0}$ ranges from 1 , where an epidemic is barely able to sustain itself, up to 18 in measles [28]. We here vary $R_{x x}$ from 1 to 10. The cross-species reproduction number $R_{y x}$ will be determined by the number of prey eaten by predators which in turn depends on their mass ratio. As the initial predator population, we similarly choose the predatorprey equilibrium value, $S_{y, 0}=\frac{\alpha \epsilon}{\phi} \frac{K \delta \nu+K \nu^{2}-\delta^{2} \epsilon-\delta \epsilon \nu}{\nu^{2} K}$, which reduces to $S_{y, 0}=\frac{\alpha \epsilon}{\phi}$ given our parameterisation.

By using this parameterisation, we are now left with only five parameters: Prey mass, predator mass, $\epsilon$, preyprey disease reproduction number, and the infection probability when predators eat infected prey. If we fix this probability at 1 , we save another parameter. This is not always a good approximation [29]. However, varying the infection probability has a much smaller effect than varying $m_{i}$ or $R_{x x}$, as is demonstrated in the supplement. We therefore choose to fix the probability at 1 . The mass parameterisation further ensures that the values of the parameters used are at least biologically plausible.

\section{Examining parameter space}

Based on the theoretical setup of our model we can make some predictions about the behaviour of the system in different regions of parameter space. Since the disease coupling constant $\beta_{x x}=\frac{R_{x x} \gamma_{x}}{\delta \epsilon} \nu=\frac{m_{x}^{1 / 2}}{700} R_{x x}$ is larger than the 
maximal predator growth rate per prey $\frac{\nu}{\epsilon}=\frac{m_{x}^{3 / 4}}{5000 m_{y}^{1 / 4}}$ for all parameter values except very high prey mass and very low predator mass, we expect that if the disease survives the initial outbreak and following depletion of susceptible prey, it will competitively exclude the predator. Given that we introduce an extinction threshhold, much of the behaviour of the system will depend on whether any of the populations reach this threshhold in the initial large excursion from equilibrium caused by the epidemic. We can derive an approximate expression determining if the disease or the predator will go extinct when prey population is initially depleted.

When hit by the epidemic, the prey population will be reduced to approximately $S_{\text {surv }} \approx S_{x, 0} e^{-R_{x x}}[2]$. We may assume that while the populations of predators and infected prey are also low, the prey will grow approximately exponentially from this low population. If $\tau_{x}$ is the time it takes for the prey to recover, this assumption gives us

$$
\begin{aligned}
S_{x} & \approx S_{x, 0} e^{-R_{x x}} e^{\alpha \tau_{x}}=S_{x, 0}, \\
\tau_{x} & \approx \frac{R_{x x}}{\alpha} .
\end{aligned}
$$

Similarly, the time $\tau_{y}$ it will take for the predator population to reach the extinction threshhold $C$ assuming exponential decay can be found as

$$
S_{y, 0} e^{-\delta \tau_{y}} \approx C \Longrightarrow \tau_{y} \approx-\frac{1}{\delta} \ln \left(\frac{C}{S_{x, 0}}\right) .
$$

By comparing eqs. (13) and (14), we find that the predator species will be able to survive the initial period of starvation if the inequality

$$
R_{x x}<-\frac{\alpha}{\delta} \ln \left(\frac{C}{S_{y, 0}}\right)=-\left(\frac{m_{y}}{m_{x}}\right)^{1 / 4} \ln \left(10^{-6} m_{y}^{3 / 4}\right)
$$

is fulfilled. An analogous expression

$$
R_{x x}<-\frac{\alpha}{\gamma} \ln \left(\frac{C}{S_{y, 0}}\right)=-\frac{7}{50} \ln \left(10^{-7} m_{x}^{3 / 4}\right)
$$

can be derived, giving the condition for the disease to survive the initial suppression of the susceptible prey population. However, since the disease growth rate is much higher than the predator growth rate, the assumption that the infected population declines exponentially until the prey population has recovered completely is no longer approximately true. This expression therefore underestimates the value of $R_{x x}$ where the transition from predator extinction to disease extinction occurs, as we shall see in fig. 1 .

Finally, since the dependence of $S_{\text {surv }}$ on $R_{x x}$ is exponential, we may assume that most prey will contract the disease in the initial oubreak, $I_{x} \approx \frac{\delta \epsilon}{\nu}$. Using this, we can derive a relation between predator mass and prey mass at the boundary where disease spillover drives the predator to extinction. The number of prey eaten must be roughly equal to the number of predators for disease spillover to become a serious threat. We therefore have that $\phi \frac{I_{x} S_{y}}{I_{x}+\epsilon} \approx S_{y}$. Given our assumption that $I_{x} \approx S_{x, 0}$ at the epidemic peak, this implies that

$$
\frac{\delta \epsilon}{\nu} \approx \frac{\epsilon}{\phi-1}
$$

By using the mass parameterisation given above, we can finally derive the relation

$$
m_{y}^{3 / 4} \approx 100 \eta m_{x}
$$

When $m_{y}^{3 / 4}>100 \eta m_{x}$, the predator is driven to extinction by disease spillover during the initial epidemic. If some portion of the prey population is immune, as we will discuss below, this mass limit becomes higher.

To test the validity of these expressions, we perform a parameter sweep where we let the different masses and the reproduction number vary logarithmically. We scan a region of parameter space large enough that the species falling within this region are interestingly different.

By inspection of numerical solutions to the model equations, we have found that after the disease outbreak, the populations will usually perform damped oscillations of initially large amplitude around some equilibrium. Although the new post-outbreak equilibria might be stable, the initial perturbation may cause the population to temporarily reach such low values that it would lead to extinction in any system with a discrete number of individuals. As initial condition, we choose the nontrivial equilibrium of eqs. (1) and (2) to avoid introducing further, artificial oscillations into the system.

We introduce an extinction threshhold of $C=10^{-5}$. If a population dips below this value, we consider it extinct. It should be noted that the precise value of the threshhold makes a relatively small difference in the end result, as might be expected due to the logarithmic relations of eqs. (15) and (16). After solving the equations numerically over $T=20000$ days, we classify the end state of the system into one of four categories: Scenarios with predator survival, disease persistence, disease-predator coexistence, and scenarios where only the healthy prey population survives. To filter out transient predator-pathogen coexistence, we let the simulation run up to $10^{5}$ days if there is still coexistence at the end of the first simulation. Plots of the regions of parameter space with predator survival and disease persistence can be seen in fig. 1.

From the plots, we see that our estimates of the behaviour of the system are approximately correct. When the epidemic does not directly affect the predators (fig. 1(a)), the predators usually survive at high $R_{x x}$. As predicted, for high prey mass, low predator mass, and high $R_{x x}$, the predator may go extinct due to starvation during the initial outbreak. We see a "zone of exclusion" at intermediate $R_{x x}$ where the disease persists, even if its upper boundary is higher (around $R_{x x}=4$ ) than predicted from eq. (16) $\left(R_{x x}=2.26\right)$. Since the disease growth rate is always higher than the predator growth rate for these parameters, the disease always drives the predator to extinction if it becomes endemic. The gap at intermediate $R_{x x}$ is thus evidence of competitive exclusion between predator 
(a)

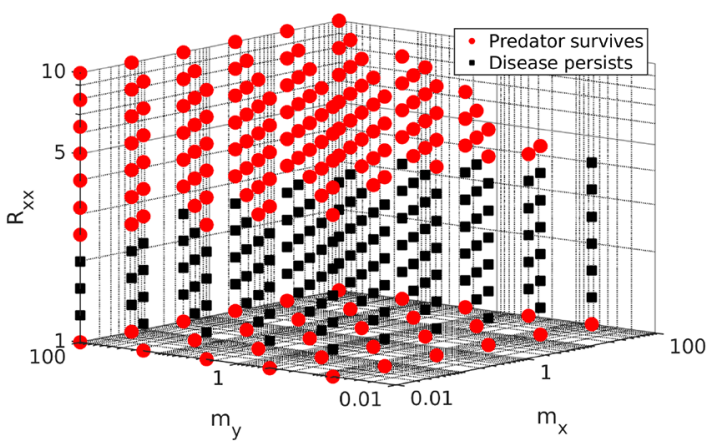

(b)
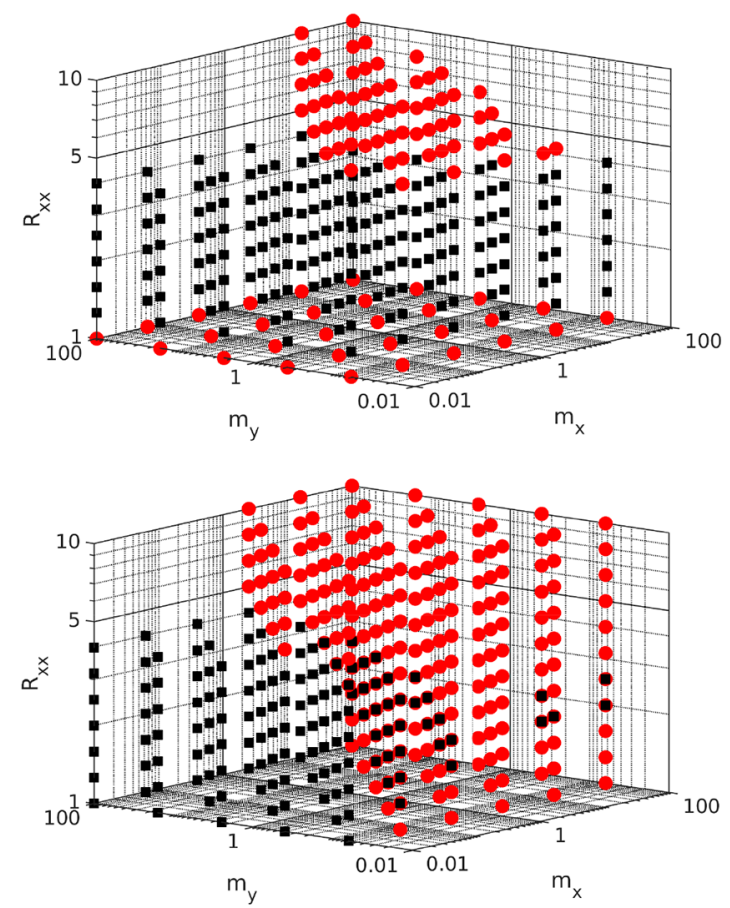

Fig. 1. Parameter space regions where the predator survives or the disease persists, as a function of prey mass $m_{x}$, predator mass $m_{y}$ (both in $\mathrm{kg}$ ), and disease reproduction number between prey $R_{x x}$. The coordinates of each red dot indicates a set of parameter values where the predator survives, while the location of the black squares indicate parameter values with disease persistence. If (a) the predators are immune, predators usually survive at high $R_{x x}$. On the other hand, if (b) the predators are susceptible, they survive at high $R_{x x}$ only if they are not too large compared to the prey. In (c), an immune prey is included alongside the susceptible one. The susceptible predators survive regardless of disease infectivity, as long as the predator is not too large compared to the prey. At low $R_{x x}$ there is predator-disease coexistence. If the predator is not susceptible, it always survives (figure not shown).

and disease, which both subsist on the same resource, the susceptible prey.

In the case where predators are susceptible to the disease (fig. 1(b)), the diagram again confirms our expectation that predators survive at high $R_{x x}$ if they have less than the predicted mass relative to the prey mass. All in all, the diagrams and our approximate calculations show that sharing a pathogen with a prey species will often cause the predator to go extinct. In fact, even an outbreak of a prey-specific epidemic can cause predator extinction, at least if the predator is a specialist.

The effect of an additional, immune prey species could be interesting to study, since this would provide the predator with a resource not shared with the pathogen. We would expect this to soften the effect of competitive exclusion. If there is disease spillover, the predator may still be driven to extinction as predicted by eq. (18), but the threshhold mass will be higher if a part of the prey population is immune. To test this hypothesis, we modify eqs. (6)-(9) to include another prey that is unaffected by the disease. We assume that the immune prey is similar to the susceptible prey and simply set their parameters to be equal. The initial combined prey population is the same as before, and the prey species do not compete. We get the results seen in fig. $1(\mathrm{c})$.

There is a striking difference compared to the case with only susceptible prey. We here see the same effect as in fig. 1(b), that predators bigger than a certain mass need to eat a lot of prey and that disease spillover therefore leads to predator extinction at high predator masses. If we instead assume that predators are immune, they always survive. At lower $R_{x x}$, the mass threshhold is not nearly as clear and cannot be easily derived through analytical arguments. The reason for this is that the system becomes chaotic in this region, which also leads to predator-disease coexistence in some cases. This has been shown and examined in more detail in a previous study [23].

To elucidate the nature of the transition between the endemic state at intermediate $R_{x x}$ and the predatordominated state at higher $R_{x x}$, we plot the local minima of the infected prey and predator population time series as functions of a control parameter in fig. 2. Figure 2(a) shows that the assumption that disease extinction is caused by a large dip in number of infected after the initial outbreak is correct. We see the global minimum value of the number of infected prey decrease with $R_{x x}$ until it reaches $10^{-5}$, at which point the disease dies out. Figure 2(b) concerns the case with two preys and examines the nature of the transition from predator exclusion to predator-disease coexistence. The figure shows two time series at different prey masses. It can be seen here how chaotic behaviour of the system may lead to disease extinction for one set of parameters and a decaying chaotic transient leads to predator-disease coexistence for another.

\section{Discussion}

The most striking conclusion to be drawn from this study is that an emerging epidemic in a specialist predator-prey system will tend to drive the predator, but not the prey, to extinction. Packer et al. have previously concluded that there are many situations in which a predator species might keep prey epidemics and parasites in check [7]. The argument that we will make based on this study is the converse: Given our dynamical model, epidemic pathogens will make life hard for predators. The parameter sweeps 

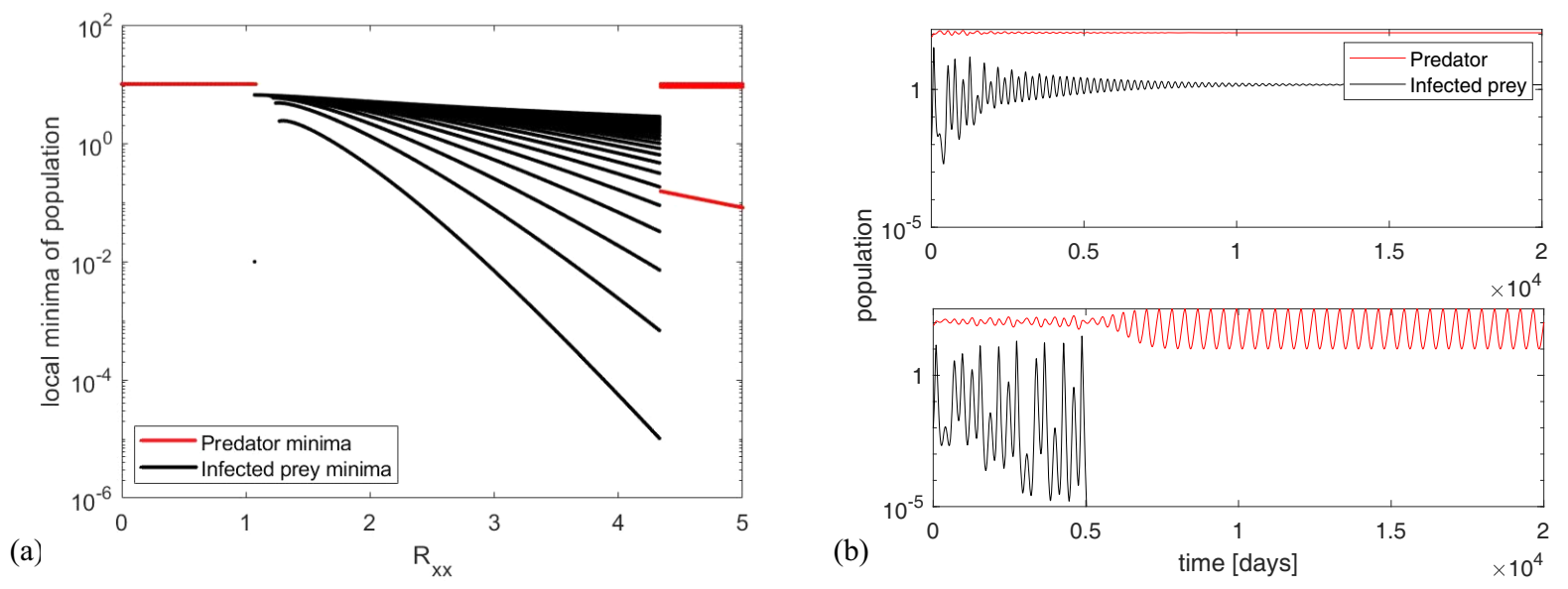

Fig. 2. Graphs illustrating the nature of transitions between different regions of parameter space. In (a) we plot the local minima of the timeseries of the infected prey population (black) and predator population (red) as a function of the control parameter $R_{x x}$. Like a bifurcation diagram, this provides information about the fixed points of the system, but also makes it possible to observe when the population reaches the extinction threshhold during an initial, large excursion. The diagram shows the transition from a predator-dominated state at low $R_{x x}$ to a disease-dominated state at intermediate $R_{x x}$, to another predatordominated state at high $R_{x x}$. The latter transition happens when the global minimum reaches the extinction threshhold, while the former happens when the predator-prey equilibrium becomes unstable at $R_{x x} \approx 1$. Here, $m_{x}=m_{y}=1$. Panel (b) shows two timeseries illustrating the transition from a state with predator-disease coexistence (upper panel) at $m_{x}=0.063$ to a predator-dominated state at $m_{x}=0.40$ (lower panel) in the version of the model with two prey species. We see that the system is at least transiently chaotic. $m_{y}=0.063$ and $R_{x x}=1.26$.

show that disease and specialist predators cannot coexist. We believe this to be an example of the disease competitively excluding the predator. Both the pathogen and the predator share a resource - the susceptible prey - and in such cases, long-term coexistence is impossible [30]. As the spread of the disease is not limited by saturation or energetic concerns, it will tend to win over the predator. What saves the predator in our model is the extinction threshhold which means that very infectious diseases do not become endemic.

The implication of this conclusion is that we should see very few ecosystems with specialist predators, prey, and a shared pathogen in the real world, as they are inherently unstable. One potential caveat is that we have here focused on mammals, using the mass scaling relations and assumptions relevant for mammalian predator-prey systems. However, due to the near-universality of massscaling relations in animals [18] we expect that most of the relations derived here should be easily transferable to other classes.

In the large region where the predator goes extinct but the pathogen becomes endemic, carrying the pathogen may still turn into an advantage for the prey species. From evolutionary biology, we know that when a pathogen becomes endemic in a given species, there will be a pressure for it to evolve to become less lethal over time [31]. This allows the pathogen to live longer in each host, and possibly to spread more effectively. An initially fatal epidemic can thus end up becoming harmless to its primary host species. If it has wiped out the predator in the process, this will represent a win-win situation for the prey species.

Finally, as an additional result, predators that are much bigger than the size of their prey are a lot more vulnerable to infection with a shared pathogen from their prey, since they need to eat more potentially infected individuals to survive. This is true even for generalist predators and is an obvious consequence if a large percentage of the prey population is infected. What is less obvious is that the upper bound on predator to prey mass ratio drops abruptly when $R_{x x}$ dips below the disease persistance threshhold in the generalist predator case. Above this threshhold, a generalist predator species can be many times the size of its infectious prey species and still not go extinct due to infection. Below the threshhold, a predator species larger than the infected prey will be driven to extinction by cross-species infections. A further complication in this region of parameter space is the emergence of chaotic behaviour, which means that within a relatively small region, there are cases where the pathogen dies out, where the predator dies out and where both coexist.

The physical reason behind the change in threshhold mass is that at high infectivities, the epidemic quickly uses up the supply of susceptibles and dies out. Therefore, a smaller portion of the predator population has time to be infected. This result, in addition to energetic concerns about hunting very small animals, could lead to an evolutionary pressure for predators to not grow too large compared to their prey.

Given all of the above, we conclude that epidemic diseases can serve as an evolutionary weapon against specialist predators. A pathogen infecting a prey species will competitively exclude any specialist predator species, even when the predator is not itself susceptible to the pathogen. Epidemics shared between predator and prey may help impose an upper limit on the predator-prey size ratio, since eating a lot of small prey is dangerous if the 
prey is infectious. The negative effect of prey disease on the predator is however weakened a lot when we take into account additional, immune prey species. The uneasy coexistence of predators and pathogens should make specialist predator-prey-disease systems rare in the real world. Our study supports the conclusion that being a specialist predator is a highly vulnerable position when the prey is affected by an epidemic pathogen, and that being a generalist should be evolutionarily favourable for predator species. Normally, one would expect that competitive exclusion presents a drive towards speciation and specialisation [32]. Our model, on the contrary, provides an example of how the inherent vulnerability of specialists will drive species towards generalisation.

In conclusion, our study supports the idea that shared epidemic diseases could be a much more important factor in the coevolution of predator and prey species than they are usually given credit for.

Our research has received funding from the European Research Council (ERC) under the European Union's Horizon 2020 research and innovation programme under grant agreement No. 740704 .

\section{Author contribution statement}

KS proposed the subject of the study and the model equations. AE produced the figures, derived the parameter space equations and wrote the manuscript. KS and $\mathrm{AE}$ revised the manuscript.

Publisher's Note The EPJ Publishers remain neutral with regard to jurisdictional claims in published maps and institutional affiliations.

Open Access This is an open access article distributed under the terms of the Creative Commons Attribution License (http://creativecommons.org/licenses/by/4.0), which permits unrestricted use, distribution, and reproduction in any medium, provided the original work is properly cited.

\section{References}

1. Mark E.J. Woolhouse, Sonya Gowtage-Sequeria, Emerg. Infect. Dis. 11, 1842 (2005).

2. Sergei Maslov, Kim Sneppen, Phys. Rev. E 96, 022412 (2017).

3. J. Chattopadhyay, O. Arino, Nonlinear Anal. Theory Methods Appl. 36, 747 (1999).
4. Herbert W. Hethcote, Wendi Wang, Litao Han, Zhien Ma, Theor. Popul. Biol. 66, 259 (2004).

5. Pierre Auger, Rachid Mchich, Tanmay Chowdhury, Gauthier Sallet, Maurice Tchuente, Joydev Chattopadhyay, J. Theor. Biol. 258, 344 (2009).

6. H.I. Freedman, Math. Biosci. 99, 143 (1990).

7. Craig Packer, Robert D. Holt, Peter J. Hudson, Kevin D. Lafferty, Andrew P. Dobson, Ecol. Lett. 6, 797 (2003).

8. Kevin D. Lafferty, Giulio DeLeo, Cheryl J. Briggs, Andrew P. Dobson, Thilo Gross, Armand M. Kuris, Science 349, 854 (2015).

9. Ying-Hen Hsieh, Chin-Kuei Hsiao, Math. Med. Biol. 25, 247 (2008).

10. Litao Han, Zhien Ma, H.W. Hethcote, Math. Comput. Modell. 34, 849 (2001).

11. Robert Henry Peters, The Ecological Implications of Body Size (Cambridge University Press, Cambridge, 1983).

12. P. Yodzis, S. Innes, Am. Nat. 139, 1151 (1992).

13. Joshua Weitz, Simon Levin, Ecol. Lett. 9, 548 (2006).

14. Andreas Eilersen, Kim Sneppen, Phys. Rev. E 99, 022405 (2019).

15. Jessica M. Cable, Brian J. Enquist, Melanie E. Moses, PLoS ONE 2, e1130 (2007).

16. A. Dobson, Am. Nat. 164, 64 (2004).

17. Geoffrey B. West, James H. Brown, Brian J. Enquist, Science 276, 122 (1997).

18. Lev Ginzburg, Mark Colyvan et al., Ecological Orbits: How Planets Move and Populations Grow (Oxford University Press on Demand, 2004).

19. Michael L. Rosenzweig, Robert H. MacArthur, Am. Nat. 97, 209 (1963).

20. Alfred J. Lotka, Proc. Natl. Acad. Sci. U.S.A. 6, 410 (1920).

21. Crawford S. Holling, Can. Entomol. 91, 385 (1959).

22. William Ogilvy Kermack, A.G. McKendrick, Gilbert Thomas Walker, Proc. R. Soc. London A 141, 94 (1933).

23. Eilersen Andreas, Mogens H. Jensen, Kim Sneppen, Sci. Rep. 10, 3907 (2020).

24. James F. Gillooly, Eric L. Charnov, Geoffrey B. West, Van M. Savage, James H. Brown, Nature 417, 70 (2002).

25. Raymond Lindeman, Ecology 23, 399 (1942).

26. P.A. Colinvaux, B.D. Barnett, Am. Nat. 114, 707 (1979).

27. Alan C. Jackson, Research advances in rabies, Advances in Virus Research, Vol. 79, 1st edition (Elsevier/Academic Press, Amsterdam Boston, 2011).

28. William J. Moss, Diane E. Griffin, Nat. Rev. Microbiol. 4, $900(2006)$.

29. R.O. Ramsden, D.H. Johnston, J. Wildl. Dis. 11, 318 (1975).

30. G.F. Gause, Science 79, 16 (1934).

31. P.W. Ewald, Emerg. Infect. Dis. 2, 245 (1996).

32. Robert Macarthur, Richard Levins, Am. Nat. 101, 377 (1967). 